\title{
Day-Ahead Self-Scheduling of Thermal Generator in Competitive Electricity Market Using Hybrid PSO
}

Pindoriya, N.M.; Singh, Sri Niwas; Østergaard, Jacob

Published in:

ISAP'09

Link to article, DOI:

10.1109/ISAP.2009.5352896

Publication date:

2009

Document Version

Publisher's PDF, also known as Version of record

Link back to DTU Orbit

Citation (APA):

Pindoriya, N. M., Singh, S. N., \& Østergaard, J. (2009). Day-Ahead Self-Scheduling of Thermal Generator in Competitive Electricity Market Using Hybrid PSO. In ISAP'09 IEEE. https://doi.org/10.1109/ISAP.2009.5352896

\section{General rights}

Copyright and moral rights for the publications made accessible in the public portal are retained by the authors and/or other copyright owners and it is a condition of accessing publications that users recognise and abide by the legal requirements associated with these rights.

- Users may download and print one copy of any publication from the public portal for the purpose of private study or research.

- You may not further distribute the material or use it for any profit-making activity or commercial gain

- You may freely distribute the URL identifying the publication in the public portal 


\title{
Day-Ahead Self-Scheduling of Thermal Generator in Competitive Electricity Market Using Hybrid PSO
}

\author{
N. M. Pindoriya, Student Member, IEEE, S. N. Singh, Senior Member, IEEE, and J. Østergaard, Member, IEEE
}

\begin{abstract}
This paper presents a hybrid particle swarm optimization algorithm (HPSO) to solve the day-ahead selfscheduling for thermal power producer in competitive electricity market. The objective functions considered to model the selfscheduling problem are: 1) to maximize the profit from selling energy in day-ahead energy market subject to operational constraints and 2) at the same time, to minimize the risk due to uncertainty in price forecast. Therefore, it is a conflicting biobjective optimization problem which has both binary and continuous optimization variables considered as constrained mixed integer nonlinear programming. To demonstrate the effectiveness of the proposed method for self-scheduling in a dayahead energy market, the locational margin price (LMP) forecast uncertainty in PJM electricity market is considered. An adaptive wavelet neural network (AWNN) is used to forecast the dayahead LMPs. The effect of risk is explicitly modeled by taking into account the estimated variance of the day-ahead LMPs.
\end{abstract}

Index Terms--Day-ahead self-scheduling, Hybrid particle swarm optimization, LMP forecast, Electricity market.

\section{INTRODUCTION}

$\mathrm{I}$ $\mathrm{N}$ a traditional power system, the unit commitment (UC) problem is solved based on the cost minimization objective while satisfying the demand and generators' operating constraints. But in a competitive electricity market, the sole objective of power producers is to maximize their profit by participating in the day-ahead and hour-ahead energy markets. Within a power pool trading, producers submit their bid (quantity with price) to the system operator (SO). For developing successful bidding strategy, power producers have to derive an optimal schedule of their generator outputs based on forecasted market clearing price. Therefore, in competitive power market environment, the conventional UC is redefined as price or profit based unit commitment (PBUC) and reported in [1]-[3]. As electrical energy price is highly volatile, future electricity prices are the main source of uncertainty faced by the producers while deriving the profit based scheduling of

N. M. Pindoriya (e-mail: naran@iitk.ac.in) is with the Department of Electrical Engineering, Indian Institute of Technology Kanpur, India. Tel: +91-512-2597009, Fax: +91-512-2590063.

S. N. Singh (e-mail: sns@elektro.dtu.dk) and J. Østergaard (e-mail: joe@elektro.dtu.dk) are with Centre for Electric Technology (CET), Department of Electrical Engineering, Denmark Technical University, Electrovej, $2800 \mathrm{Kgs}$. Lyngby, Denmark. S. N. Singh is on leave from the Department of Electrical Engineering, Indian Institute of Technology Kanpur, India. their generators. Therefore, the self-scheduling for power producers is a conflicting bi-objective optimization problem where producer wishes to maximize his profit and to minimize the risk associated with forecasted price uncertainty while satisfying all operational constraints simultaneously.

The generation self-scheduling problem needs to determine two types of decision variables. The unit operating status (on/off), which is binary variable $(1 / 0)$ and the power generated by the unit, is a continuous variable. Therefore, it can be considered as two kinds of combined constraint optimization problems, namely optimal commitment (on/off) and economic generation dispatch of the units during the scheduling period (one day -one week).

Thus, generation self-scheduling problem belongs to the class of nonlinear programming-hard problem and is very complex. A new category of heuristic optimization tools has emerged to cope with some of the traditional optimization algorithms' shortcomings. The main heuristic optimization techniques are genetic algorithm (GA), evolutionary programming (EP), and particle swarm optimizer (PSO). These have been successfully applied to a wide range of power system optimization problems in which the conventional optimization is not applicable or difficult to find the global optimal solution. Compared to other heuristic optimization techniques, PSO has been found to be robust for solving problem featuring non-linearity, non-differentiability, multiple optima and high dimensionality through adaptation.

Within the context of the day-ahead self-scheduling for thermal power producer, the problem has been formulated as mixed integer quadratic programming (MIQP) problem [4][5]. The hybrid techniques that combine lagrangian relaxation (LR) and other heuristic optimization techniques such as GA, EP and PSO, to solve the lagrangian augmented objective function for the risk based self-scheduling problem has been proposed in [6].

This paper proposes hybrid PSO (HPSO) to handle both types of decision variables (continuous and binary), through combination of original PSO and binary PSO. The locational marginal price (LMP) forecast uncertainty in PJM electricity market is considered to implicitly include the uncertainty related to congestions. The adaptive wavelet neural network (AWNN) model [7] is used to forecast the day-ahead LMPs. The power producer considered in this paper is assumed to be a price-taker; therefore, the self-scheduling of each generator 
is independent of the self-scheduling of the others. For simplicity, a power producer having a single generator is considered in this paper. However, the proposed approach can be extended for several generators also.

\section{PROBLEM FormULATION}

The objective of self-scheduling problem for power producer is to maximize his expected profit and to minimize the risk simultaneously, while satisfying all generating unit constraints over the scheduling period. The profit of the producer for the scheduling period, $\mathrm{T}$, is the expected revenue minus total operating cost. The expected revenue from the sales of energy in a day-ahead market is the forecasted market price times the actual power production in each hourly time period. The total operating cost includes the production cost of the scheduled combination units and the transition cost. The production cost at any given time interval is assumed to be quadratic function of generator power output. The transition cost is the sum of the start-up cost and shut-down cost, which is associated with unit on/off to off/on transitions. It is a complex mathematical optimization problem with both integer and continuous variables. The self-scheduling optimization problem for single generator can be formulated as [8]:

(1) Maximize the expected profit

$$
F_{1}\left(P_{G, t}, u_{t}, \lambda_{t}^{f o r}\right)=\left\{\begin{array}{l}
\underbrace{\sum_{t=1}^{T} \lambda_{t}^{\text {for }}\left(P_{G, t}\right) u_{t}-}_{\text {Revenue }} \\
\underbrace{\sum_{t=1}^{T}\left(a+b P_{G, t}+c P_{G, t}^{2}\right) u_{t}}_{\text {Production cost }} \\
\sum_{\text {Transition cost }}^{\sum_{t=1}^{T}\left[\begin{array}{l}
S U C_{t}\left(1-u_{t-1}\right) u_{t} \\
+S D C_{t}\left(1-u_{t}\right) u_{t-1}
\end{array}\right]}
\end{array}\right\}
$$

(2) Minimize the risk

$$
F_{2}\left(P_{G, t}, u_{t}, V^{e s t}\right)=\sum_{i=1}^{T} \sum_{j=1}^{T}\left[V_{i j}^{e s t} \times\left(P_{G, i} u_{i}\right)\left(P_{G, j} u_{j}\right)\right]
$$

where,

$\begin{array}{ll}\lambda_{t}^{\text {for }} & \text { forecasted price at hour } t \\ V^{\text {est }} & \text { estimated covariance matrix } \\ P_{G, t} & \text { power generated at hour } t \\ u_{t} & \text { schedule state of thermal unit at hour } t \\ & \text { (1: unit is on and 0: unit is off) } \\ S U C_{t} & \text { start-up cost at hour } t \\ S D C_{t} & \text { shut down cost at hour } t \\ a, b, c & \text { generating unit cost coefficients }\end{array}$

The start-up cost for restarting a decommited generating unit depends on the off time of unit prior to start-up, $T_{O F F}$.
This paper considered the exponential cost curve (3) for the detailed modeling of start-up cost, and the fixed shut-down cost.

$$
S U C_{t}=\chi+\delta\left[1-\exp \left(\frac{-T_{O F F, t-1}}{\gamma}\right)\right]
$$

where,
$\chi$
$\delta$
$\gamma \quad$ unit cooling time constant
$T_{\text {ON,t }} / T_{\text {OFF, } t} \quad$ continuously on/off time of unit up to hour $t$

The most common measure of risk is variance or its square root i.e., standard deviation. The effect of risk is explicitly modeled by taking into account the estimated variance of the LMPs. In (2), the covariance matrix, $V^{\text {est }}$, is $T \times T$ matrix and it can be estimated based on available actual and forecasted price for the last $D$ days, using the following exponentially weighted moving average equation [9].

$$
V^{e s t}=(1-\alpha) \sum_{i=1}^{D} \alpha^{i-1}\left(\Lambda_{D-i+1}^{a c t}-\Lambda_{D-i+1}^{f o r}\right)\left(\Lambda_{D-i+1}^{a c t}-\Lambda_{D-i+1}^{f o r}\right)^{\prime}
$$

In (4), the parameter $\alpha(0<\alpha<1)$ is decay factor, $D$ is greater than or equal to 24 to ensure the covariance matrix positive definite, and $\Lambda=\left[\lambda_{1}, \ldots, \lambda_{T}\right]^{\prime}$. This paper considered the generation schedule for next day to participate in dayahead energy market, therefore the scheduling period, $T=24$.

The self-scheduling problem using these two conflicting objective functions $\left(F_{1}\right.$ and $\left.F_{2}\right)$ is converted to a single objective function with the help of risk tolerance parameter $\beta$. The resulting problem formulation becomes

$$
\operatorname{Maximize}\left[F_{1}\left(P_{G, t}, u_{t}, \lambda_{t}^{f o r}\right)-\beta \times F_{2}\left(P_{G, t}, u_{t}, V^{e s t}\right)\right]
$$

while satisfying the following constraints:

- Generator operating limit:

$P_{G}^{\min } u_{t} \leq P_{G} \leq P_{G}^{\max } u_{t} ; \quad t=1,2, \ldots, T$

- Ramp up/down limit:

Because of physical restriction on thermal generating unit, the generation may increase or decrease with corresponding rampup and ramp-down rate limits. So unit is constrained due to the ramp rate limit as mentioned below.

If power generation increases, $P_{G, t}-P_{G, t-1} \leq R U$

If power generation decreases, $P_{G, t-1}-P_{G, t} \leq R D$

Therefore, the generating unit output is limited by rampup/down rate at each hour and hence, the operating limits given in (6) are modified as follow:

$$
\left.\begin{array}{l}
\max \left[P_{G}^{\min },\left(P_{G, t-1}-R D\right)\right] \leq P_{G, t} \\
\min \left[P_{G}^{\max },\left(P_{G, t-1}+R U\right)\right] \geq P_{G, t}
\end{array}\right\}
$$


- Minimum up/down-time limit:

Once the unit is committed/decommitted, there must be a minimum time before it can be decommitted/committed.

$\left.\begin{array}{l}{\left[\left(u_{t}-u_{t-1}\right)\left(T_{O N, t-1}-M U T\right)\right] \leq 0} \\ {\left[\left(u_{t}-u_{t-1}\right)\left(T_{O F F, t-1}-M D T\right)\right] \geq 0}\end{array}\right\}$

where, the time counter for which a unit has been on/off at hour $t, T_{O N, t} / T_{O F F, t}$ can be expressed as:

$\left.\begin{array}{l}T_{O N, t}=\left(1+T_{O N, t-1}\right) u_{t} \\ T_{O F, t}=\left(1+T_{O F F, t-1}\right)\left(1-u_{t}\right)\end{array}\right\}$

where,

$P_{G}^{\min } / P_{G}^{\max } \quad$ minimum and maximum generation limits

$R U / R D \quad$ ramp up/down limits

$M U T / M D T \quad$ minimum up/down time limits

Satisfying the load demand is no longer an obligation in self-scheduling for power producer in the competitive electricity market, therefore, it is not considered, in this paper, as a constraint. The risk tolerance parameter, $\beta$, depends on the preference of the power producer. The risk seeking producer looking for higher profit chooses low (close to zero) value for $\beta$; on the contrary, risk adverse producer chooses large value for $\beta$ to increase the risk measure in (5).

\section{PARTICLE SWARM OPTIMIZATION (PSO)}

\section{A. Original PSO}

Particle swarm optimization (PSO), first, proposed by Kennedy and Eberhart [10] is a population-based stochastic optimization technique inspired by social behavior of organisms such as bird flocking and/or fish schooling. Particle swarm algorithm starts with the random initialization of a population of potential solutions called particles in the multidimensional search space and particles fly around in a search space to approach the optima. During flight, the individual particles are attracted stochastically toward the positions of their own best fitness achieved so far and the best fitness achieved so far by any of their neighbours. Thus, a PSO technique controls the balance between local and global exploration of the problem space and that helps to overcome premature convergence and also enhanced the searching ability. Compared to other optimization techniques, a PSO has been found to be robust for solving problem featuring nonlinearity, non-differentiability, multiple optima and high dimensionality through adaptation.

Suppose that the search space is $D$-dimensional, then the $i$ th particle of the population (i.e. position) can be represented by a $D$-dimensional vector, $X_{i}=\left[x_{i 1}, x_{i 2}, \ldots, x_{i D}\right]$. The velocity (position change) of this particle can be represented by another $D$-dimensional vector, $V_{i}=\left[v_{i 1}, v_{i 2}, \ldots, v_{i D}\right]$. Each particle keeps track of its coordinates in the search space which are associated with the best solution achieved so far and is called pbest . The best previous position of $i$-th particle is recorded and represented as pbest. Defining the index of the best particle among all the particles in the swarm is represented by the gbest. The modified velocity and position of each particle can be calculated using the current velocity and the distance from pbest to gbest as shown in the following equations:

$$
\begin{aligned}
& v_{i d}^{k+1}=w \times v_{i d}^{k}+c_{1} R_{1}\left(\text { pbest }_{i d}-x_{i d}^{k}\right)+c_{2} R_{2}\left(\text { gbest }_{d}-x_{i d}^{k}\right) \\
& x_{i d}^{k+1}=x_{i d}^{k}+v_{i d}^{k+1}
\end{aligned}
$$

where, $d=1,2, \ldots, D, i=1,2, \ldots, N$ and $N$ is the size of the swarm; $c_{1}$ and $c_{2}$ are two positive acceleration coefficients which keep balance between the particle's individual and social behavior. $R_{1}$ and $R_{2}$ are uniformly distributed random numbers in $[0,1]$ added in the model to introduce stochastic nature. The inertia weight of the particle, $w$, is suitably selected to control the exploration properties of the algorithm. Exploration requires bigger step size at the beginning of the optimization process to determine the most promising area (global search) then the step size is reduced to focus only on that area (local search). Therefore, $w$, is linearly decreasing function of the iteration index, iter as given in (12).

$$
w=w_{\max }-\frac{w_{\max }-w_{\min }}{\text { iter }_{\max }} \times \text { iter }
$$

where, $w_{\max }$ and $w_{\min }$ are the initial and final weights, respectively, whereas, iter ${ }_{\max }$ and iter is the maximum number of iterations and the current iteration number, respectively.

Besides, a maximum allowable velocity vector, $v_{\max }$, clamps velocities of particles on each dimension. If the acceleration causes the velocity on that dimension to exceed $v_{\max }$ specified by the user, then the velocity on that dimension will be limited to $v_{\max }$. Generally, Vmax is set at $10-20 \%$ of the dynamic range of the variable on each dimension.

\section{B. Binary PSO}

The original version of PSO discussed in Section III.A basically developed for continuous optimization problems. The binary PSO [11] is made possible with a simple modification to original version of PSO. This BPSO solve the binary problems similar to those traditionally optimized by genetic algorithms. In BPSO, $x_{i}$ and pbest can take on value of 1 or 0 only. The velocity $v_{i}$ will determine a probability of threshold using logistic functions as follows:

$\operatorname{Pr}\left(v_{i}\right)=\frac{1}{1+\exp \left(-v_{i}\right)}$

A random number (drawn from a uniform distribution in $[0.0,1.0])$ is then generated, whereby $x_{i}$ is set to 1 if the random number is less than the value from the sigmoid function (13) as illustrated below:

$$
x_{i}= \begin{cases}1, & \text { if rand }()<\operatorname{Pr}\left(v_{i}\right) \\ 0, & \text { otherwise }\end{cases}
$$


Thus real velocity is digitized (1/0) by logistic functions (13 and 14) for binary space.

\section{HYBRID PSO (HPSO) FOR SELF-SCHEDULING}

In this section implementation of HPSO to solve selfscheduling problem for thermal generator is explained. The generation scheduling problem needs to determine two types of decision variables. The unit operating status (on/off), u which is a binary variable $(1 / 0)$ and the power generated by the unit, PG which is a continuous variable. Therefore, it can be considered as two kinds of combined constraint optimization problems, namely optimal commitment (on/off) and economic generation dispatch of the units during the scheduling period, $T$. Among these two, first is non-linear optimization problem and second is binary optimization problem. This paper considered original PSO along with BPSO to solve generation scheduling optimization problem.

\section{A. Particle Formulation}

Before applying PSO to solve generation scheduling problem, the representation of a particle must be formulated. Since, this paper considered the generation schedule for next day to participate in day-ahead energy market, there are 24 continuous variables representing power generation and 24 binary variables for operating status (on/off) of the unit, for each hours of the next day. The continuous variables are initialized with uniformly distributed pseudorandom numbers that take the range of these variables, i.e., $P_{G}=\operatorname{rand}\left[P_{G}^{\min }, P_{G}^{\max }\right]$. However, in the case of the binary variables, an additional operator is needed to account for the distinct nature of these variables and those are initialized using (13 and 14) from randomly initialized velocity. Once the binary variables are initialized, those should check for the feasibility (the minimum up-down constraints). Hence, finally, each string having the first twenty four are real valued particles and next twenty four (i.e., 25-48) are binary particles, and can be represented as

$$
P_{i}=[\underbrace{P_{G, 1}, P_{G, 2}, \ldots, P_{G, T}}_{\text {Continuous }}, \underbrace{u_{1}, u_{1}, \ldots, u_{T}}_{\text {Binary }}]
$$

For example, if power producer having a single generator and population size in PSO is $L$, then dimension of population is equal to be $L \times 48$.

\section{B. Function Evaluation and Constraints Handling}

The quality of an individual of the population is found using fitness function evaluation. After formulating and randomly initializing the particles in a feasible solution space, each individual string will be evaluated using evaluation function (5). Based on fitness values, pbest and gbest will be calculated. The velocity of whole string is updated using (10) and if any updated velocity is violating its upper and lower limits $\left(v_{\max }\right.$ and $\left.v_{\min }\right)$ then the velocity is set to the violated limit. Then, the position of particles corresponding to continuous variables and binary variables are modified using
(11) and (14), respectively. After that all variables should go through a feasibility check to satisfy the all operational constraints. Handling the constraints relating to the problem is a major factor in the application of PSO approaches to the self scheduling optimization.

There are different ways to handle constraints in heuristic optimization algorithms just like in the case of the PSO. The following constraint handling methods are the most commonly used ones [12].

1. Preserving feasible solution method: In this method, solutions are initially placed in a feasible search space and remain within by adapting an update mechanism that generates only feasible solutions.

2. Infeasible solution rejection method: This approach rejects any solution that violates the feasible search space.

3. Penalty function method: Here, a penalty factor is added to the objective once any constraint violation occurs.

4. Solution repair method: This approach converts the infeasible solution to a feasible one by performing special operations.

Within the context of PSO applications to the selfscheduling, inequality constraints representing the permissible power generation, $P_{G}$, variables are typically handled through set to limit approach (SLA). If $P_{G}$ violates its upper or lower bound, obtained from (7), the value of this variable is set to the violated limit. By this way, the non-linear constraints corresponding to generator operating limits including ramp limits have been handled. The minimum up/down time constraints (8), also require the enforcement of condition usually formulated as nonlinear constraints. These constraints may be violated from the obtained generation schedule in the each iteration. Therefore, these will be checked and repaired if the violations occur. The process of fixing a solution is done by evaluating the state of unit $T_{O N, t}$ and $T_{O F F, t}$. For the first hour of the scheduling period (at $t=1$ ), unit is assumed to be ON for last MUT-1 hours and therefore, it has to be $\mathrm{ON}$ at this hour to satisfy the minimum up time constraint. The procedure for minimum up-and down time repair method suggested in [13] has been used in this paper.

\section{CASE STUdy}

To demonstrate the effectiveness of the proposed HPSO based technique for day-ahead generation scheduling, this paper considers the case study of power producer owning a single generating unit in PECO control zone of PJM electricity market [14]. The generator data are given in Tables I and II. The shut-down cost for the generator is assumed to be zero. The AWNN based price forecasting model [7] has been used to forecast the day-ahead LMPs in the same control zone of PJM market for the year 2007. The AWNN is a new class of feed-forward neural network having continuous wavelet function as activation functions of the hidden layer nodes. Therefore, it combines the time-frequency localization characteristic of wavelet and learning ability of feed-forward neural network into a single unit. The forecasted prices are provided in Table III. The covariance matrix is estimated 
using (4) based on actual and forecasted LMP data for the last 24 days just prior to the day of the estimation day. This paper takes $\alpha=0.99$ and $D=24$ for the covariance matrix estimation.

TABLE I

GENERATOR TECHNiCAL PARAMETERS

\begin{tabular}{|c|c|c|c|c|c|}
\hline $\begin{array}{c}P_{G}^{\min } \\
(\mathrm{MW})\end{array}$ & $\begin{array}{c}P_{G}^{\max } \\
(\mathrm{MW})\end{array}$ & $\begin{array}{c}\mathrm{RU} \\
(\mathrm{MW} / \mathrm{h})\end{array}$ & $\begin{array}{c}\mathrm{RD} \\
(\mathrm{MW} / \mathrm{h})\end{array}$ & $\begin{array}{c}\mathrm{MUT} \\
\text { (hours) }\end{array}$ & $\begin{array}{c}\mathrm{MDT} \\
\text { (hours) }\end{array}$ \\
\hline 150 & 455 & 130 & 130 & 4 & 4 \\
\hline
\end{tabular}

TABLE II

GeNERATOR COST CHARACTERISTIC

\begin{tabular}{|c|c|c|c|c|c|}
\hline \multicolumn{3}{|c|}{ Fuel cost } & \multicolumn{3}{c|}{ Start-up cost } \\
\hline$a$ & $b$ & $c$ & $\chi$ & $\delta$ & $\gamma$ \\
\hline 1000 & 16.19 & 0.00048 & 4500 & 4500 & 4 \\
\hline
\end{tabular}

TABLE III

FORECASTED LMP

\begin{tabular}{|c|c|c|c|c|c|}
\hline Hour & $\begin{array}{c}\text { LMP } \\
(\$ / M W h)\end{array}$ & Hour & $\begin{array}{c}\text { LMP } \\
(\$ / M W h)\end{array}$ & Hour & $\begin{array}{c}\text { LMP } \\
(\$ / M W h)\end{array}$ \\
\hline 1 & 21.48 & 9 & 49.26 & 17 & 73.12 \\
\hline 2 & 19.53 & 10 & 57.46 & 18 & 72.48 \\
\hline 3 & 19.56 & 11 & 60.02 & 19 & 67.76 \\
\hline 4 & 19.44 & 12 & 68.98 & 20 & 62.92 \\
\hline 5 & 21.09 & 13 & 69.17 & 21 & 69.97 \\
\hline 6 & 23.46 & 14 & 72.45 & 22 & 69.50 \\
\hline 7 & 28.47 & 15 & 71.43 & 23 & 43.64 \\
\hline 8 & 42.11 & 16 & 68.63 & 24 & 32.70 \\
\hline
\end{tabular}

Although the HPSO method seems to be sensitive to the tuning of some weights and/or parameters, after number of simulation trails, the HPSO parameters of proposed method are set as follows:

- Population size $=40$;

- Initial inertia weight $\left(w_{\max }\right)=0.9$;

- Final inertia weight $\left(w_{\min }\right)=0.4$;

- Maximum Iterations $=2000$;

- Acceleration constant $c_{1}=2$ and $c_{2}=2$;

- $v_{\max }=2.0$ and $v_{\min }=-2.0$;

In order to overcome the stochastic nature of the HPSO for obtaining better result, 10 runs are averaged, starting with different random initial population particles.

The self-scheduling problem given by (5) is solved for different values of the risk tolerance parameter, $\beta$, which allows assigning the different risk level of the power producer in self-scheduling. The efficient frontier which depicts expected profit versus risk of the power producer is illustrated in Fig. 1. It is observed that expected profit increases as risk level of the generator increases. Fig. 2 shows the generation schedule of power producer for the case of maximum risk $(\beta=$ $0)$ and minimum risk $(\beta=0.05)$. It can be seen from Fig. 2 that, the generating unit of risk-seeking power producer with maximum risk $(\beta=0)$ is $\mathrm{ON}$ for the entire scheduling period, whereas risk-averse producer $(\beta=0.05)$ is ON for fewer hours than risk seeking generator.
It is also noted that the risk seeking generator is $\mathrm{ON}$ for entire scheduling period with maximum power output of the unit (i.e., $455 \mathrm{MW}$ ) except hours 1-3, where the profit is less because of low LMP for that period. In hour 1, although the profit of risk-averse generator is very less (because low LMP), unit cannot be shut-down due to the minimum up time constraints, because it is assumed that unit to be $\mathrm{ON}$ for last MUT-1 hours. It should also be noted that for risk-averse generator with minimum risk $(\beta=0.05)$, there is reduction in power output during on-peak period (hours 14-19). This power output reduction because of the generator is facing high risk in that period due to price forecast uncertainty.

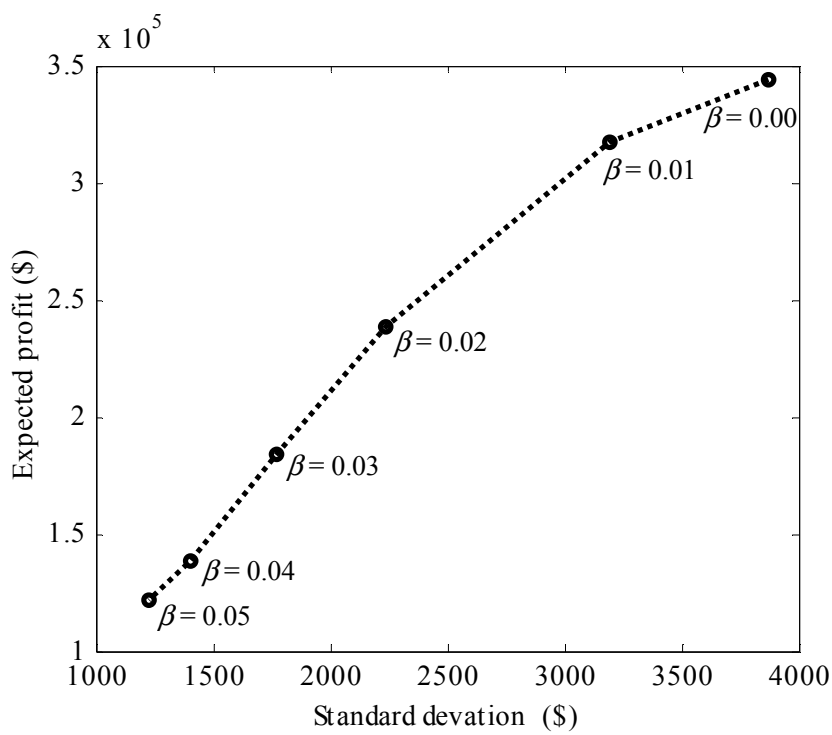

Fig. 1. Efficient frontier

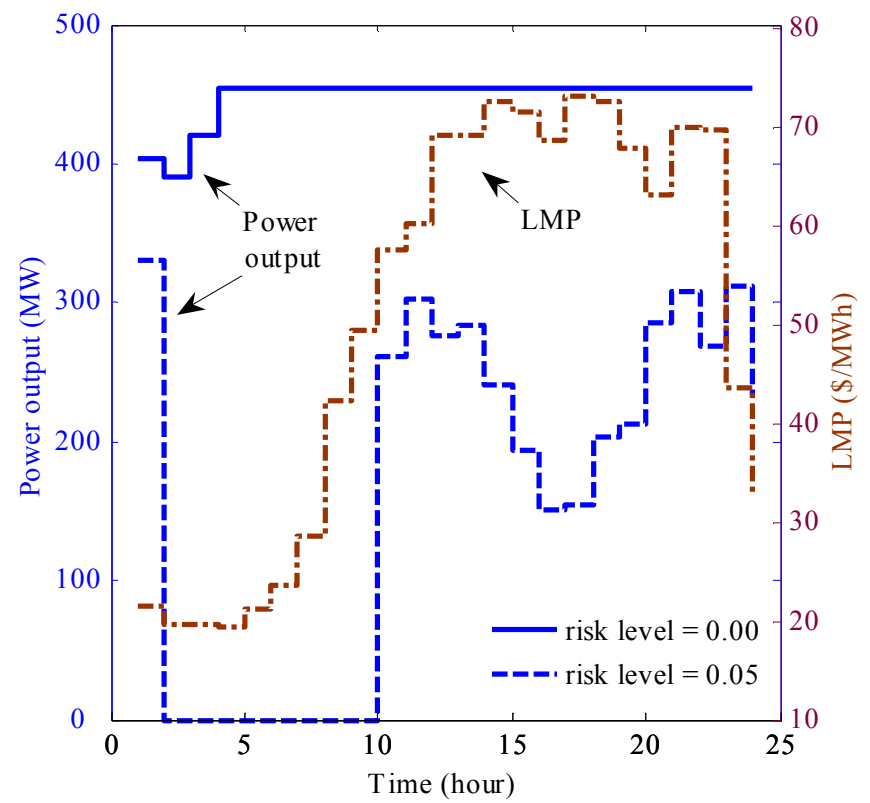

Fig. 2. Hourly generation schedule

\section{CONCLUSION}

Self-scheduling of power producer in a day-ahead energy market is a conflicting bi-objective optimization problem which has both binary and continuous optimization variables 
considered as constrained mixed integer nonlinear programming. The self-scheduling problem for thermal generator is addressed, in this paper, considering maximizing the profit and at the same time, minimizing the risk due to uncertainty in price forecast. The hybrid particle swarm optimization (HPSO), which is the combination of the original PSO and binary PSO, has been applied to solve the constrained mixed integer nonlinear problem. The selfscheduling results for the different risk levels of power producer in PJM electricity market have been reported in this paper. It is found that expected profit increases as risk level of the generator increases.

\section{REFERENCES}

[1] M. Shahidehpour, H. Yamin, and Z. Li, Market operations in electric power systems: forecasting, scheduling, and risk management, New York: Wiley, 2002.

[2] Tao Li and Mohammad Shahidehpour, "Price-based unit commitment: A case of lagrangian relaxation versus mixed integer programming," IEEE Trans. Power Syst., vol. 20, no. 4, pp. 2015-2025, Nov. 2005.

[3] C. W. Richter and G. B. Sheble, "A profit based unit commitment GA for the competitive environment," IEEE Trans. Power Syst., vol. 15, no. 2, pp. 715-721, May 2000.

[4] H. Yamin and M. Shahidehpour, "Risk and profit in self scheduling for Gencos," IEEE Trans. Power Syst., vol. 19, no. 4, pp. 2104-2106, Nov. 2004.

[5] J. Conejo, F. J. Nogales, J. M. Arroyo, and R. García-Bertrand, "Riskconstrained self-scheduling of a thermal power producer," IEEE Trans. Power Syst., vol. 19, no. 3, pp. 1569-1574, Aug. 2004.

[6] H.Y. Yamin, I.A. Altawil, et al., "Risk based self scheduling for Gencos in the day-ahead competitive electricity markets," European Trans. Electrical Power, 2008.

[7] N. M. Pindoriya, S. N. Singh, and S. K. Singh, "An adaptive wavelet neural network based energy price forecasting in electricity markets," IEEE Trans. Power Syst., vol.23, no.3, pp.1423-1432, Aug. 2008.

[8] N. M. Pindoriya and S. N. Singh, "MOPSO based day-ahead optimal self-scheduling of generators under electricity price forecast uncertainty," Proc. IEEE PEG General Meeting, July 26-30, 2009, Calgary, AB, Canada.
[9] J. P. Morgan, RiskMetrics-Technical Document, $4^{\text {th }}$ Ed. New York: Morgan Guaranty Trust Company, 1996.

[10] J. Kennedy and R. Eberhart, "Particle swarm optimization," Proc. of IEEE Conf. Neural Networks, 1995, pp.1942-1948.

[11] J. Kennedy and R. Eberhart, "A discrete binary version of the particle swarm optimization algorithm," Proc. of the 1997 conf. Systems, Man, and Cybernetics, 1997, pp. 4104-4109.

[12] G. Coath and S. K. Halgamuge, "A comparison of constraint-handling methods for the application of particle swarm optimization to constrained nonlinear optimization problems," Proc. Congr. Evolutionary Computation, Australia, vol. 4, pp. 2419-2425, 2003.

[13] V.N. Dieu and W. Ongsakul, "Enhanced augmented Lagrangian Hopfield network for unit commitment," IEE Proc., Gener. Transm. Distrib., vol. 153, no. 6, pp. 624-632, 2006.

[14] PJM Electricity market website. [Online]. http://www.pjm.com/.

\section{BIOGRAPHIES}

N. M. Pindoriya (S'07) obtained his M. E. Degree in Electrical Engineering from M. S. University, Baroda in 2002. He worked as Lecturer in Electrical Engineering Department at CIT-Changa, Anand, India from 2003-2005, and presently pursuing his Ph.D. in the Department of Electrical Engineering, Indian Institute of Technology Kanpur, India. His research interests are deregulated power systems and economics, ANN applications to forecast electricity market variables, and application of PSO in power system optimization.

S. N. Singh (SM'02) received M. Tech. and Ph.D. from Indian Institute of Technology Kanpur, India in 1989 and 1995, respectively. Presently, he is visiting professor in the Department of Electrical Engineering, Denmark Technical University, Denmark, and on leave from the Department of Electrical Engineering, Indian Institute of Technology Kanpur, India. His research interests include power system restructuring, power system optimization \& control, voltage security and stability analysis, power system planning, and ANN application to power system problems. He is a Fellow of IE (India), Fellow of IETE (India), member IET (UK) and senior member of IEEE.

Jacob Østergaard (M'95) received MSc. from Denmark Technical University in 2005. Presently he is Professor and Head of the Centre for Electric Technology, in the Department of Electrical Engineering, Technical University of Denmark. His research interests include integration of renewable energy, control architecture for future power system, and demand side. Professor Østergaard is serving in several professional organizations including the EU Smart Grids advisory council. 